\title{
Reducing lumbar spine flexion using real-time biofeedback during patient handling tasks
}

\author{
Mohammadhasan Owlia ${ }^{\mathrm{a}, \mathrm{b}}$, Megan Kamachi ${ }^{\mathrm{a}, \mathrm{c}}$ and Tilak Dutta ${ }^{\mathrm{a}, \mathrm{c}, *}$ \\ ${ }^{a}$ Toronto Rehabilitation Institute, University Health Network, ON, Canada \\ ${ }^{\mathrm{b}}$ Department of Mechanical and Industrial Engineering, University of Toronto, ON, Canada \\ ${ }^{\mathrm{c}}$ Institute of Biomaterials and Biomedical Engineering, University of Toronto, ON, Canada
}

Received 6 March 2019

Accepted 6 August 2019

\begin{abstract}
.
BACKGROUND: Patient handling activities require caregivers to adopt postures that increase the risk of back injury. Training programs relying primarily on didactic methods have been shown to be ineffective in reducing this risk. The use of real-time biofeedback has potential as an alternative training method.

OBJECTIVE: To investigate the effect of real-time biofeedback on time spent by caregivers in end-range lumbar spine flexion.

METHODS: Novice participants were divided into intervention $(n=10)$ and control $(n=10)$ groups and were asked to perform a set of simulated care activities eight times on two consecutive days. Individuals in the intervention group watched a training video on safer movement strategies and received real-time auditory feedback from a wearable device (PostureCoach) in four training trials whenever their lumbar spine flexion exceeded a threshold (70\% of maximum flexion). Changes in end-range lumbar spine flexion were compared between groups and across trials.

RESULTS: Participants in the intervention group saw reductions in end-range lumbar spine flexion during the simulated patient handling tasks at the end of the training compared to their baseline trials while there was no change for the control group.

CONCLUSIONS: The training program including PostureCoach has the potential to help caregivers learn to use safer postures that reduce the risk of back injury.
\end{abstract}

Keywords: PostureCoach, personal support workers, family caregivers, low back pain, multi-component training intervention

\section{Introduction}

Home caregivers suffer from low back injuries at high rates [1, 2]. Many of these back injuries are likely the result of patient handling tasks. These tasks

\footnotetext{
*Address for correspondence: Tilak Dutta, 550 University Ave., Room 11-131, Toronto, ON, M5G 2A2, Canada. Tel.: +1 416597 3422/Ext 7956; E-mail: tilak.dutta@uhn.ca.
}

often require caregivers to adopt awkward postures while supporting heavy loads (during activities such as bathing and toileting) [3-6]. More than eight million Canadians provide care to individuals at home for chronic health conditions, disability or age-related needs [7]. The aging population [8], as well as the trend of shifting patient care from hospitals to home, will continue to increase the burden on home care- 
givers [9]. The majority of care at home is provided by unpaid caregivers, while only supplementary assistance is provided by paid home support workers [10].

Caregiver injuries result in large economic costs, as well as personal costs to those who suffer from the resulting pain [11]. Rates of musculoskeletal disorders among U.S. healthcare workers are much higher than the rate for the average worker [12]. Healthcare workers are injured more than workers in many industries that are traditionally considered high risk such as construction, or maintenance and repair [12]. Over the past 10 years in Ontario, the low back has consistently been the most frequently injured body part among occupations related to support of health services [13].

In keeping with the recent trend in ergonomics, efforts to "fit the task to the worker" [14] have seen reductions in injury risk resulting from the widespread introduction of patient lifting devices in hospitals and nursing homes [15-18]. However, community-based caregivers do not typically have access to mechanical lift devices [19]. They also commonly work alone [20-23], in cramped and cluttered spaces [21, 23-27], with limited training [28]. These workers are routinely asked to perform tasks that would not be allowed in hospitals or nursing homes, where existing policies make manual patient handling impermissible. Instead, all patient handling in institutional care requires the use of a mechanical lift device operated by two care providers $[29,30]$.

In contrast, caregivers in the home routinely perform tasks such as patient lifting, repositioning, bathing and toileting, which require manually supporting heavy loads in awkward postures [5, 31]. Interventions that reduce exposure to these heavy care tasks result in caregivers reporting less pain [32].

Studies have reported that the burden of low back pain (LBP) is higher than for any other disability [33, 34]. Back pain can be predicted by factors including the magnitude and frequency of trunk flexion, time spent in a forward flexed trunk or non-neutral postures, and trunk movements with high angular velocity [35-38]. In particular, it has been shown that repetitive end-range lumbar spine flexion leads to disc damage [32, 39-41]. For instance, porcine model in vitro studies using porcine vertebrae have shown that end-range spine flexion can increase the risk of injury because the flexed spine segments are weaker than neutral spine segments [42].

The majority of efforts to reduce the risk of back injury among caregivers over the past decades have been focused on "fitting tasks to the worker" by redesigning tasks as is the trend in the field [14]. However, many caregiving activities in the home environment, such as bathing and toileting patients, have not been restructured. Therefore, we believe there is an urgent need to make the most of any other strategies that may be available for preventing home caregiver injuries. In his review paper, McGill (2009) notes the field of ergonomics has focused on "fitting the task to the work" in the last few decades which has had a positive effect on injury rates [43]. $\mathrm{He}$ argues it is now time for the ergonomics community to turn its focus back to "fitting the worker to the task" because there is likely much unrealized benefit to be exploited. This is particularly relevant to homecare workers where both the care tasks and the work environment are difficult to change.

In particular, McGill advocates for retraining movement patterns and suggests a number of movement-based back injury prevention strategies [43]. He points out that it is particularly important to train individuals to avoid postures where the spine is flexed to reduce the risk of low back injury. Instead, workers should be trained to bend using a "hip hinge", which allows the individual to maintain the natural curve in the lower back (lumbar lordosis). Indeed, a posture assessment study of home health care workers found elevated musculoskeletal injury risks in those workers who stood with their trunk bent forward, likely with the spine flexed near the end of its range [31].

\subsection{Traditional back safety training}

Conventional training sessions provided to caregivers are typically didactic lecture-based presentations that may be supplemented with a short practice session [44, 45]. These training sessions typically include discussion of directives like "lift with your legs, not with you back" in an attempt to train workers to avoid spine flexion [46]. However, this type of training has been found to be ineffective at reducing the risk of injury [47-52]. Perhaps these results should come as no surprise considering the shocking lack of evidence supporting how the actual training methods that are deployed. This lack of evidence represents a massive opportunity to reduce the risk of injury considering how widespread use of this type of training is across industries. For instance, Beach et al. (2018) showed that simply changing a directive from "lift without rounding your lower back" had a greater effect on reducing spine flexion than 
when participants were directed to "lift with your legs instead of your back" or "bend your knees and hips" [46]. Similarly, there has been little work to determine if predominantly didactic (lecture style) methods are appropriate for training skills that require motor learning [53].

A further problem with existing training methods is that it can be difficult to provide training that reflects the nature of the challenges caregivers face in the home. Care tasks can vary dramatically depending on the particular client and the environment [61]. Most real-world care tasks cannot be completed following the idealized conditions that are portrayed in training sessions [44]. Caregivers are often left to come up with their own approaches in the field, which may undervalue the importance of minimizing the risk of injury in favour of other factors. These challenges may explain why $39 \%$ of Ontario personal support workers said they lacked the training they needed to perform care tasks they were assigned [62].

It is important to note that some researchers would argue there is an alternative hypothesis to explain why current training interventions have been ineffective: Spine flexion does not lead to negative outcomes for those without a history of back pain/injury [54]. Some call for an end to movement-based training that targets reductions in spine flexion $[55,56]$. They argue that back pain and bulging discs are a natural part of aging [57] and unavoidable. There is a particular focus on pointing out that we should not be limiting spine flexion in pain free individuals $[55,58]$.

While this is a reasonable hypothesis that needs further testing, we believe that the existing epidemiological and biomechanical evidence $[39,59,60]$ also points to an equally reasonable hypothesis: reducing flexion can indeed reduce the risk of back pain/injury but existing training methods are not able to shift workers to reduce spine flexion long-term [61]. For instance, one study on musculoskeletal injuries in hospitals found that behaviour modification was difficult to achieve and, even if it was achieved, new behaviors were often short lived [44]. We argue that before we can expect to see a change in pain/ injury risk for caregivers, we need to see a change in behaviour demonstrating a given training program resulted in motor learning.

The training program evaluated in this study consisted of two components: i) a training video designed to provide caregivers basic back injury prevention knowledge and ii) real-time feedback from PostureCoach to support motor learning resulting in reduced lumbar spine flexion.

\subsection{Augmented feedback in training}

The use of augmented feedback is a powerful approach for achieving behaviour change. There is a rich body of research on the use of this approach in the field of motor learning often for applications involving coaching of athletes. We argue caregivers should be treated more like "occupational athletes" to help highlight the importance of applying carefully practiced techniques and movements on-the-job to project against injury [63]. However, since it is impractical to provide each caregiver a coach to work with in the field, we consider whether a wearable device may be able to provide some augmented feedback (coaching) that would help shift workers to safer patterns of movement.

Systems for delivering movement-centred feedback typically include: (1) sensors to measure movement or posture; (2) a computer or microcontroller to process the signal; and (3) and some method to communicate feedback to the user [64]. Examples of movement-centred feedback uses include: correcting slouching behavior [65], adolescent scoliosis therapy [66], reducing warehouse working injury rates [67], Parkinson's disease therapy [68], nonspecific chronic low back pain therapy [69] and a video game designed to improve lifting technique [70]. Sensors used in these systems include mechanical switches [65], electromagnetic trackers [67], strain gauges [71], and accelerometers [72]. Feedback is provided with either auditory $[65-68,72]$ or vibrotactile actuators [69]. To ensure improvement in performance, the timeliness of feedback has been shown to be an important factor to consider, with realtime feedback demonstrating the best performance [73].

We have developed PostureCoach, a wearable device for providing real-time feedback based on lumbar spine flexion. This study aims to evaluate the effectiveness of a training program, consisting of a short training video as well as training sessions with Posture Coach, for novice caregivers performing simulated care tasks. We hypothesized that participants exposed to our training program would reduce their end-range (80th and 95th percentile) lumbar spine flexion.

\section{Material and methods}

\subsection{Participants}

Twenty healthy adults with no formal training in caregiving or patient handling were recruited for this 
Table 1

Demographic information of the participants recruited for this study. Mean $\pm \mathrm{SD}$ values are reported, where applicable

\begin{tabular}{lcc}
\hline & Control $(n=10)$ & Intervention $(n=10)$ \\
\hline Age (years) & $24.7 \pm 2.7$ & $28.1 \pm 6.4$ \\
Sex & 6 female; 4 male & 4 female; 6 male \\
Height $(\mathrm{cm})$ & $172.1 \pm 8.3$ & $171.6 \pm 7.2$ \\
Mass $(\mathrm{kg})$ & $62.8 \pm 10.2$ & $71.3 \pm 16.3$ \\
\hline
\end{tabular}

study (Table 1). We chose this population for this initial study with our training program because we are likely to see the greatest effect with novices based on our own work [74] as well as the motor learning literature [75-77]. For instance, it is likely we need to rely on different strategies when helping experienced caregivers unlearn bad motor habits. Our participants were individuals who may need to take on the role of providing care for friends or family members as novice unpaid caregivers.

Participants were randomly assigned to the experimental group $(n=10)$ or control group $(n=10)$. Participants were recruited from the Toronto Rehabilitation Institute and University of Toronto communities. All participants were over the age of 18, and could speak and understand English. They had no history of back pain in the last six months and had no musculoskeletal issues related to the spine. The study protocol was approved by the University
Health Network Research Ethics Board prior to the start of this study.

\subsection{Setting and instrumentation}

The setting for this study was a simulated home environment (HomeLab at Toronto Rehabilitation Institute), which consisted of a furnished bedroom, living room, bathroom and kitchen. This space resembled a typical single-story house with functioning wiring and plumbing (Fig. 1). A member of research team (24-year-old female, $179 \mathrm{~cm}$ in height and $83 \mathrm{~kg}$ in mass) performed the role of patient actor in all trials. The patient actor was instructed to play the role of a frail older adult who was able to partially bear weight when standing, but was unsteady because of poor balance control. A wheelchair (NRG+, Maple Leaf Wheelchair Mfg Inc., Mississauga, ON) with adjustable armrests, push brakes and swing out standard footrests was used in this study.

All participants were asked to don PostureCoach and remain wearing the device for the duration of all trials. PostureCoach consisted of a pair of MTi-3 (Xsens Technologies, Enschede, Netherlands) inertial measurement units (IMUs). As shown in Fig. 2, the upper sensor was positioned near T10 using adjustable straps and the lower one was positioned

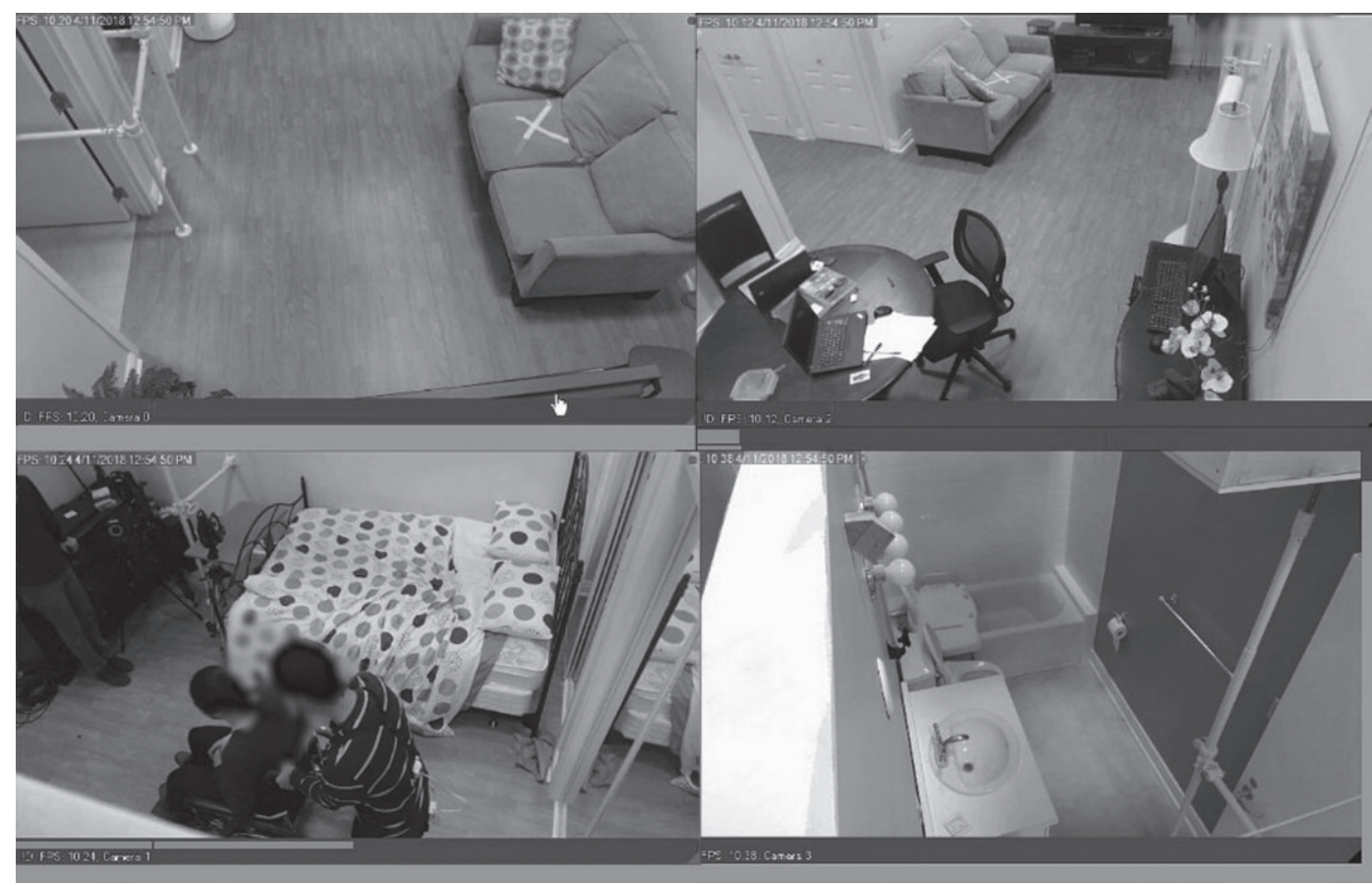

Fig. 1. Setup of HomeLab for this study. 


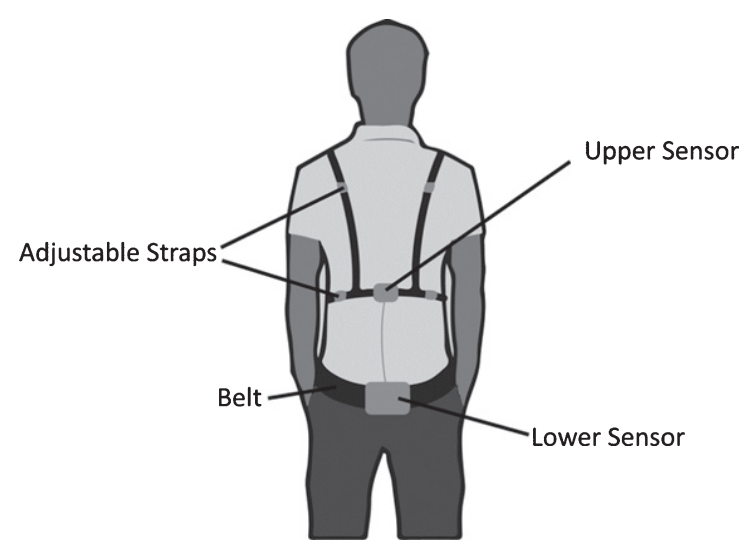

Fig. 2. Setup of PostureCoach.

near the sacrum, secured with a sacroiliac belt (Serola Biomechanics, Inc., Loves Park, IL). An M3 32-bit microcontroller was used for reading the sensors' output and for determining their relative orientations of the upper and lower IMUs. The relative angle between the two sensors was decomposed based on the orientation of lower sensor. Finally, the angle between the two sensors in the sagittal plane was used to represent the amount of lumbar spine flexion.

The system provided audible feedback to be delivered when the spine flexion angle exceeded a predefined threshold. The output of both sensors was recorded on memory card. A validation study with this system showed that it was able to track sagittal lumbar spine flexion with RMS error of $2.3^{\circ}$ and $r=0.986$ (Pearson correlation coefficient) when compared to a Vicon motion capture system [78].

For all participants, maximum forward flexion was measured using a toe-touch activity. In intervention trials, PostureCoach produced a continuous audible tone based on the spine flexion threshold angle equal to $70 \%$ of each participant's maximum forward lumbar flexion angle (70\% max). The threshold of 70\% was chosen because it is known that beyond this value extensor moments produced by tissues proximal to the spine begin to increase exponentially [79]. A second threshold was set to the lumbar spine flexion angle equal to $20^{\circ}$ less than the $70 \%$ max value, which was used to provide intermittent tones that increased in frequency, up to the $70 \%$ max threshold, at which point the sound became a continuous tone. The intermittent tones were added after pilot testing with PostureCoach showed that the sudden onset of the continuous tone at the $70 \%$ of maximum flexion threshold had the potential to startle some participants.

\subsection{Procedure}

A member of research team was present to guide participants through each data collection trial shown in Fig. 3. The study consisted of two one-hour long sessions on consecutive days. Participants performed the same set of care tasks four times on each day.

\subsubsection{Session one}

After obtaining written consent, a member of research team walked each participant through a typical series of caregiving tasks and addressed any questions or concerns. Following this introduction, participants were asked to don PostureCoach and bend over and touch their toes to record each person's maximum spine flexion angle followed by the series of care tasks with the patient actor (Trial 1). This value was used to set the auditory feedback threshold (70\% of max flexion), as well for normalizing all lumbar spine flexion data recorded by PostureCoach to account for differences in sensor placement between the two testing sessions. This max flexion value was also recorded at a number of times during each session to correct for shifting of either sensor during the sessions.

Next, participants in the intervention group watched a training video with information about safe patient handling strategies including information about why it is important to avoid lumbar spine flexion and how this can be achieved with the hip hinge technique. This training video (youtu.be/vtDGheJlfh4) was developed in collaboration with Occupational Health \& Wellness team of SE Health. The points discussed in the video were adapted from existing literature [43, 78, 79]. Control participants did not watch the training video. Next, participants were asked to repeat the set of simulated care tasks again (Trial 2). Feedback was disabled from PostureCoach during Trial 1 and Trial 2.

Intervention group participants were then asked to perform the caregiving tasks twice more (Trial 3 and Trial 4), with PostureCoach's real-time feedback enabled (the control group still had feedback disabled).

\subsubsection{Session two}

On the following day, participants again performed the same simulated care activities without feedback, to measure baseline performance during this session (Trial 5). Afterwards participants repeated the care activities again two more times (Trial 6 and Trial 7), but with feedback turned on for the intervention group 


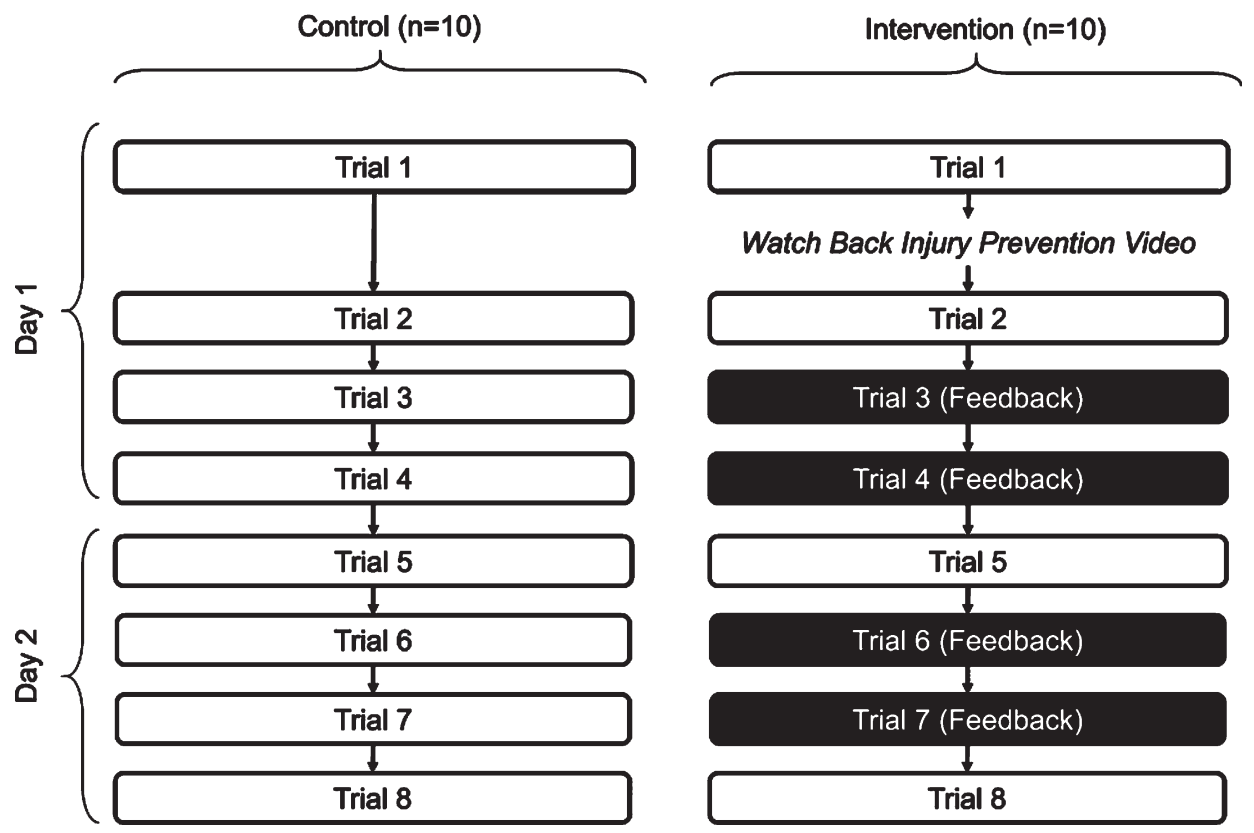

Fig. 3. Schematic of the updated protocol used in this study.

and feedback turned off for those in the control group. After a short break, they again performed the care tasks (Trial 8), without feedback in both groups, in order to determine whether the use of PostureCoach resulted in a short-term behavior change.

\subsection{Caregiving tasks}

In each trial, the participants were asked to complete the following series of simulated care activities, on the patient actor with a counterbalanced order:

- Wheeling the wheelchair from the living room to the bedroom

- Transferring the patient from the bed to the wheelchair

- Wheeling the patient to the living room and placing the wheelchair near the couch

- Transferring the patient to the couch

- Transferring the patient from the couch to wheelchair

- Wheeling the patient from THE living room to THE bathroom

- Helping the patient to stand, doff his/her pants, and sit on the toilet

- Helping the patient to stand, don his/her pants, and sit on the wheelchair

- Wheeling the patient back to the bedroom and preparing the bed
- Transferring the patient from the wheelchair to the bed

- Moving the wheelchair back to the living room

Participants were asked to make sure that brakes were engaged and footrests were out of the way during all patient transfer activities.

\subsection{Data analysis}

The spine flexion angle data was normalized by calculating the median of all the max flexion values recorded over the eight trials. This allowed for expressing each participant's data as a percentage of max flexion. This normalization helped to eliminate differences due to variations in sensor placement, as well as account for differences between participants' height and flexibility.

Next, the data was trimmed by removing the max flexion measurements and the remaining data was used to create histograms of lumbar spine flexion angles for each trial. The 50th, 80th and 95th percentiles of spine flexion were also calculated.

A mixed design analysis of variance (ANOVA) with one between-subject variable (control or intervention groups) and one within-subject variable of trial number (Trial 1, Trial 2, Trial 5, Trial 8) was used to investigate the differences between groups. Note, 
the chosen trials did not have feedback provided for participants in either group.

Bonferroni post-hoc tests were used to investigate differences in the 50th, 80th and 95th percentiles of spine flexion between Trial 1 and Trial 2 (video effect) as well as BETWEEN Trial 1 and Trial 8 (program effect) in intervention group. Independent $t$-tests were used for comparing measures of distribution (percentiles) between groups, in Trial 1 and Trial 8 .

\section{Results}

Figure 4 shows the histogram of the percent of maximum lumbar spine flexion angle for all participants in the intervention group for Trials 1 and 8 . Figure 5 shows the same histogram for all participants in the control group. Recall none of the participants in either group received feedback from PostureCoach in these trials.

The 50th, 80th and 95th percentile values of spine flexion for control and intervention group are shown in Fig. 6.

Mauchly's test of sphericity showed no violations of sphericity in any of the measures (percentiles) for the mixed ANOVA. There was a significant main effect of trial number across all three measures, with $F=(3,54)=9.179, p<0.0005, \eta_{p}^{2}=$ $0.365, F=(3,54)=10,232, \quad p<0.0005, \eta_{p}^{2}=0.390$, and $F=(3,54)=10.082, p<0.005, \eta_{p}^{2}=0.387$ for 50th, 80th and 95th percentile, respectively.

Moreover, there was also an interaction effect between trial and group for 80th and 95th percentile of forward flexion, with $F=(3,54)=9.348$, $p<0.0005, \quad \eta_{p}^{2}=0.369$, and $F=(3,54)=11.779$, $p<0.0005, \eta_{p}^{2}=0.424$ respectively.

Post-hoc tests (Bonferroni) revealed that while there were no significant differences in the 50th or 80th percentile of forward flexion values between trials 1 and 2 for individuals in intervention group, there was a significant difference in the 95th percentile value of flexion $(p=0.05)$, shown in Fig. 7 . Post-hoc tests also revealed that the 80th and 95th percentile forward flexion values in Trial 1 were significantly higher than Trial 8 among intervention group ( $p=0.24$ and $p=0.002$, respectively), while there were no significant differences for the control group (Fig. 8).

\section{Discussion}

The histogram shown in Fig. 4 demonstrates that participants in the intervention group shifted to using safer postures over the two-day training period. We can see the histogram shifts to the left as participants spend less time in end-range spine flexion if we compare Trial 1 to Trial 8. This demonstrates that participants in the intervention group changed their

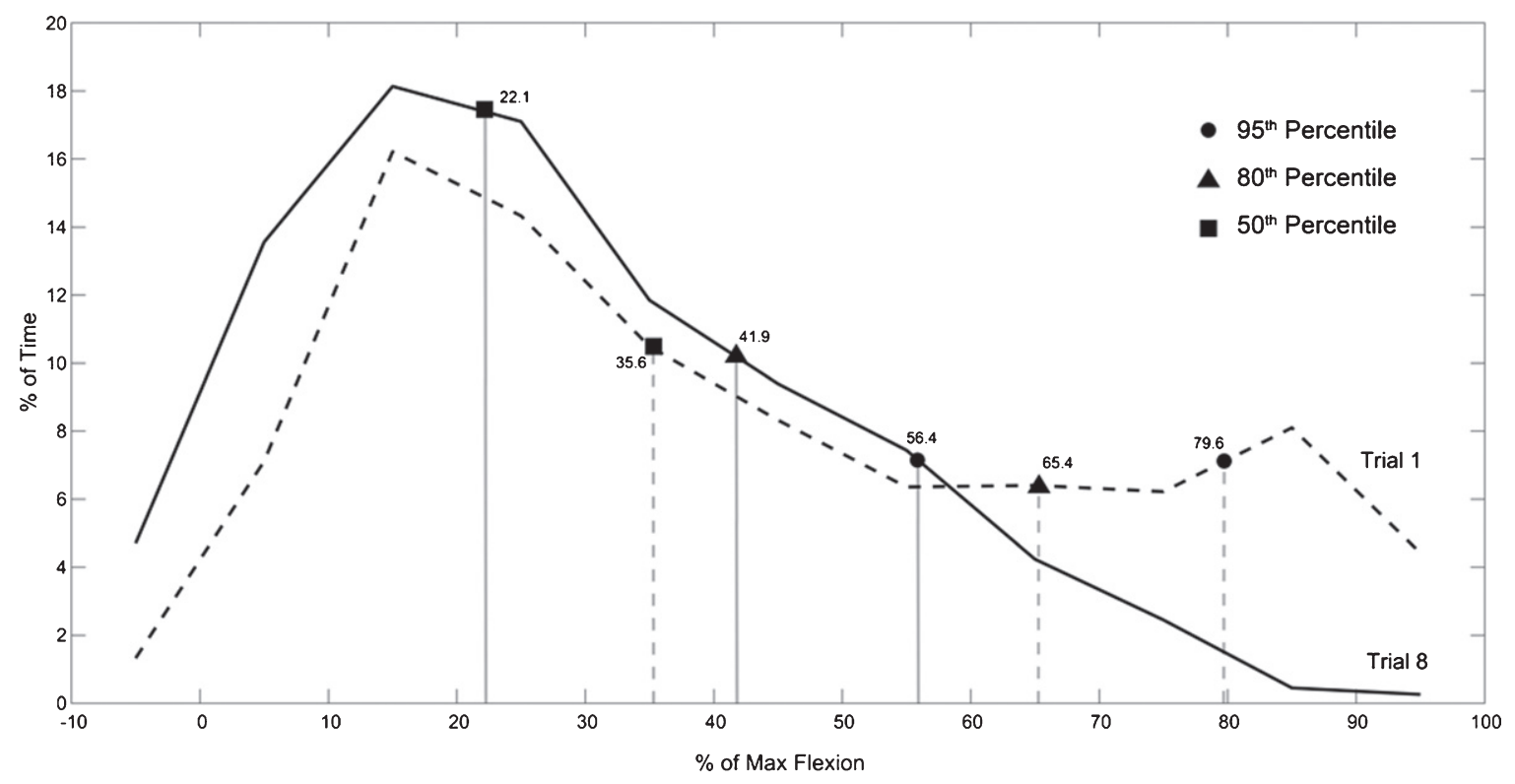

Fig. 4. Average histogram of forward spine flexion angles (in degrees) in intervention group for Trial 1, and Trial 8. 


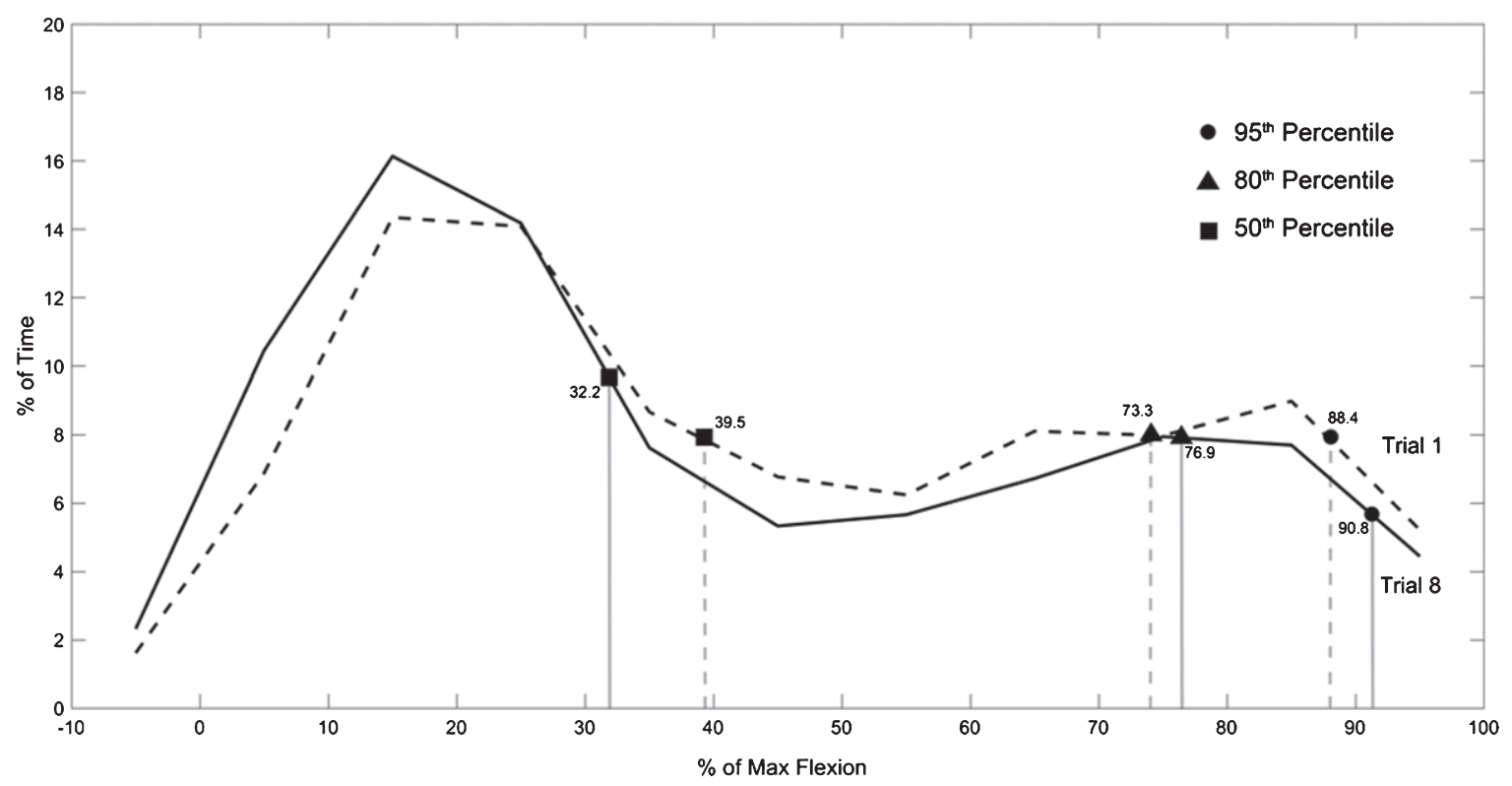

Fig. 5. Average histogram of forward spine flexion angles (in degrees) in control group for Trial 1, and Trial 8.

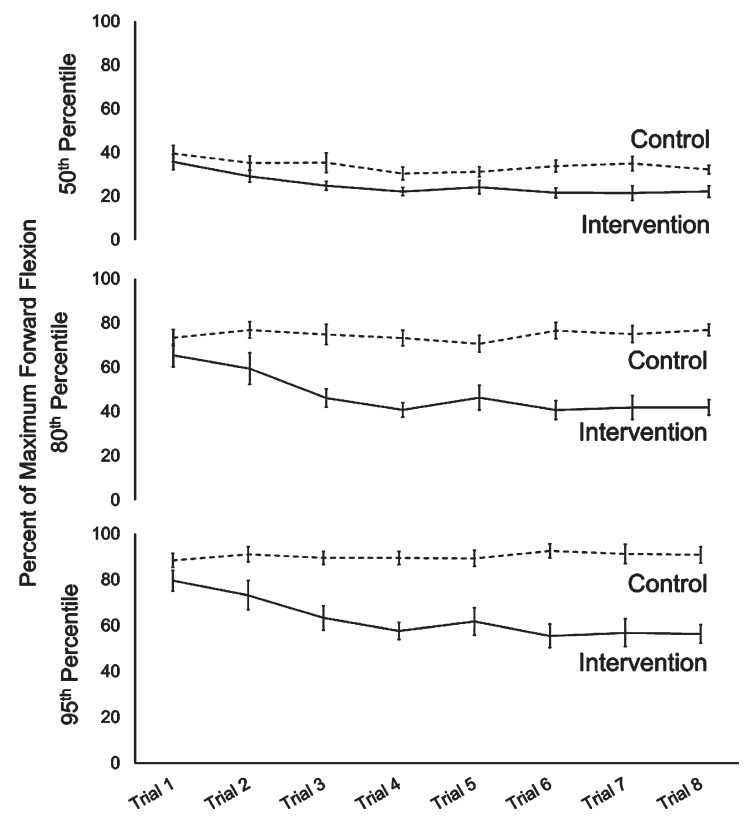

Fig. 6. 50th, 80th and 95 th percentile of forward flexion/maximum flexion across trials in intervention and control groups. Error bars show standard error.

behaviour and spent less time in end-range spine flexion as a result of the training program. We can also see that there little difference between the histograms comparing Trial 1 and Trial 8 for the control group in Fig. 5.

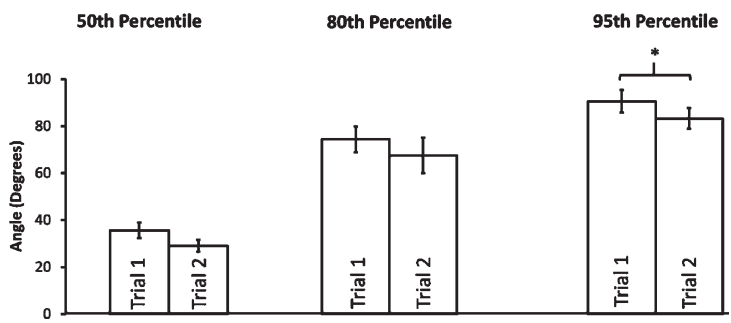

Fig. 7. Video effect among individuals in intervention group. Error bars show standard error.

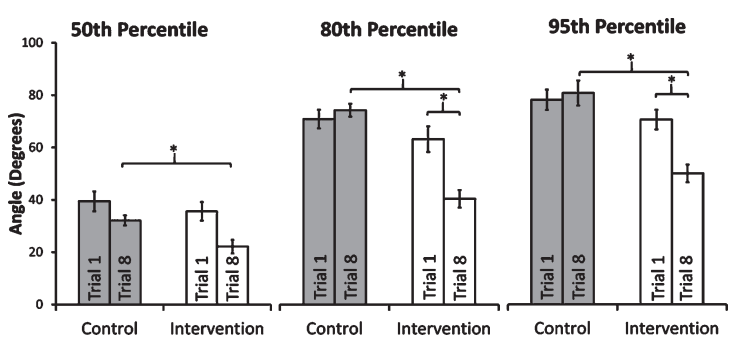

Fig. 8. Changes in 50th, 80th and 95th percentile lumbar spine flexion values between Trial 1 and Trial 8 for the intervention and control groups. Error bars show standard error.

Figure 8 shows that the median value (50th percentile) of forward flexion remained statistically unchanged in both groups between Trial 1 and Trial 8 . However, the 80th and 95th percentile flexion values decreased in the intervention group, but not in the control group. The 80th and 95th percentile flexion 
values in the intervention group were reduced by $36 \%$ and $29 \%$, respectively.

Finally, our training video had a small statistically significant effect on the 95th percentile forward flexion values (8\% reduction) among participants in the intervention group, while 50th and 80th percentile values remained the same between Trial 1 and Trial 2.

These findings suggest that both the video and realtime feedback from PostureCoach may be valuable to include in back injury prevention training programs for caregivers. Future work will evaluate the long-term retention of changes in behaviour and the potential for this training to be used in the field with real caregivers as they deliver care [80]. The ultimate goal is to determine whether this approach can also reduce injury rates.

Finally, there were three limitations with this study that may have had an impact on the findings or the potential for the findings to be applied elsewhere:

- The participant population was made up of individuals who were likely to have more knowledge about back injury prevention than a true novice caregiver.

- HomeLab is an idealized environment and most homes are not as clutter-free and accessible.

- The patient actor in the study was cooperative, which does not induce any psychological or emotional stressors that could have effected motor learning.

- The belt (attached to the lower sensor) would shift on occasion during data-collection sessions. The study coordinator would readjust the belt when this happened but it is likely the lower sensor was not in optimal position for short periods.

\section{Conclusion}

In this study, we investigated the effectiveness of a back injury prevention training program including a video and real-time feedback from PostureCoach to reduce end-range spine flexion for novice caregivers. We found that the training program was able to reduce 80th and 95th percentile spine flexion values by $36 \%$ and $29 \%$, respectively while performing a series of simulated care activities after a two-day training period. Therefore, this training program has the potential to help caregivers who perform patient care tasks in the home environment.

\section{Acknowledgments}

This work was made possible by funding from the AGE-WELL, supported by the Government of Canada through the Networks of Centres of Excellence program. The views expressed do not necessarily reflect those of these organizations.

\section{Conflict of interest}

None to report.

\section{References}

[1] Wood EM, Hegmann KT, Garg A, Alder SC, Thiese MS, Thompson C, editors. Back, neck, and shoulder pain in home healthcare workers. 1 st Annual Regional National Occupational Research Agenda (NORA) Young/New Investigators Symposium; 2003.

[2] US Bureau of Labor Statistics. Nonfatal Occupational Injuries and Illnesses Requiring Days Away From Work, 2015. [Internet]. 2016. Available from: https://www.bls.gov/news.release/pdf/osh2.pdf.

[3] Howard N, Adams D. An analysis of injuries among home health care workers using the Washington state workers' compensation claims database. Home Health Care Services Quarterly. 2010;29(2):55-74.

[4] Lum JM, Sladek J, Ying A. Home support workers in the continuum of care for older people: Canadian Research Network for Care in the Community; 2015.

[5] King EC, Boscart VM, Weiss BM, Dutta T, Callaghan JP, Fernie GR. Assisting frail seniors with toileting in a home bathroom: approaches used by home care providers. Journal of Applied Gerontology. 2019;38(5):717-49.

[6] King EC, Weiss BM, Boscart VM, Dutta T, Callaghan JP, Fernie GR. Bathing frail seniors at home: Home care providers' approaches. WORK: A journal of Prevention, Assessment and Rehabilitation, in review. 2019.

[7] Sinha M, Bleakney A. Receiving care at home: Statistics Canada; 2014.

[8] Statistics Canada. Population Projections for Canada (2013 to 2063), Provinces and Territories (2013 to 2038). 2015.

[9] Keefe JM, Fancey PJ, Ellis T, Hawkins G. A portrait of unpaid care in Nova Scotia: Healthy Balance Research Program Halifax (NS); 2006.

[10] Lilly MB. Medical versus social work-places: constructing and compensating the personal support worker across health care settings in Ontario, Canada. Gender, Place and Culture. 2008;15(3):285-99.

[11] Mock C, Cherian MN. The global burden of musculoskeletal injuries: challenges and solutions. Clinical Orthopaedics and Related Research. 2008;466(10):2306.

[12] US Bureau of Labor Statistics. Nonfatal occupational injuries and illnesses requiring days away from work, 2015. 2016.

[13] Board WSI. By the Numbers: WSIB Statistical Report 2017.

[14] Grandjean E, Kroemer KH. Fitting the task to the human: a textbook of occupational ergonomics: CRC press; 1997.

[15] Collins JW, Wolf L, Bell J, Evanoff B. An evaluation of a "best practices" musculoskeletal injury prevention program in nursing homes. Injury Prevention. 2004;10(4):206-11. 
[16] Li J, Wolf L, Evanoff B. Use of mechanical patient lifts decreased musculoskeletal symptoms and injuries among health care workers. Injury Prevention. 2004;10(4):212-6.

[17] Trinkoff AM, Brady B, Nielsen K. Workplace prevention and musculoskeletal injuries in nurses. Journal of Nursing Administration. 2003;33(3):153-8.

[18] Nagavarapu S, Lavender SA, Marras WS. Spine loading during the application and removal of lifting slings: the effects of patient weight, bed height and work method. Ergonomics. 2017;60(5):636-48.

[19] Brigham CJ. Safe handling of residents in home health care. Ergonomics in Design. 2010;18(1):26-8.

[20] Lang A, Macdonald MT, Storch J, Stevenson L, Mitchell $\mathrm{L}$, Barber T, et al. Researching triads in home care: perceptions of safety from home care clients, their caregivers, and providers. Home Health Care Management \& Practice. 2014;26(2):59-71.

[21] Markkanen P, Quinn M, Galligan C, Sama S, Brouillette N, Okyere D. Characterizing the nature of home care work and occupational hazards: a developmental intervention study. American Journal of Industrial Medicine. 2014;57(4):44557.

[22] Giosa J, Holyoke P, Bender D, Tudge S, Gifford W. Observe, coach, assist, and report: An emerging framework for integrating unregulated healthcare providers into interdisciplinary healthcare teams. Journal of Research in Interprofessional Practice and Education. 2015;5(2).

[23] Craven C, Byrne K, Sims-Gould J, Martin-Matthews A. Types and patterns of safety concerns in home care: staff perspectives. International Journal for Quality in Health Care. 2012;24(5):525-31.

[24] Polivka BJ, Wills CE, Darragh A, Lavender S, Sommerich C, Stredney D. Environmental health and safety hazards experienced by home health care providers: a room-by-room analysis. Workplace health \& safety. 2015;63(11):512-22.

[25] Hignett S, Otter ME, Keen C. Safety risks associated with physical interactions between patients and caregivers during treatment and care delivery in Home Care settings: A systematic review. International Journal of Nursing Studies. 2016;59:1-14

[26] Dutta T, Holliday PJ, Gorski SM, Baharvandy MS, Fernie GR. The effects of caregiver experience on low back loads during floor and overhead lift maneuvering activities. International Journal of Industrial Ergonomics. 2011;41(6):653-60.

[27] Markkanen P, Quinn M, Galligan C, Chalupka S, Davis L, Laramie A. There's no place like home: a qualitative study of the working conditions of home health care providers. Journal of Occupational and Environmental Medicine. 2007;49(3):327-37.

[28] Carneiro P, Martins J, Torres M. Musculoskeletal disorder risk assessment in home care nurses. Work. 2015;51(4):65765.

[29] Occupational Safety and Health Administration. Guidelines for Nursing Homes: Ergonomics for the Prevention of Musculoskeletal Disorder. 2009.

[30] Waters TR. When is it safe to manually lift a patient? AJN The American Journal of Nursing. 2007;107(8):53-8.

[31] Brulin C, Gerdle B, Granlund B, Höög J, Knutson A, Sundelin G. Physical and psychosocial work-related risk factors associated with musculoskeletal symptoms among home care personnel. Scandinavian Journal of Caring Sciences. 1998;12(2):104-10.

[32] Czuba LR, Sommerich CM, Lavender SA. Ergonomic and safety risk factors in home health care: Explo- ration and assessment of alternative interventions. Work. 2012;42(3):341-53

[33] Storheim K, Zwart JA. Musculoskeletal disorders and the Global Burden of Disease study. BMJ Publishing Group Ltd; 2014.

[34] Hoy D, March L, Brooks P, Blyth F, Woolf A, Bain C, et al. The global burden of low back pain: estimates from the Global Burden of Disease 2010 study. Annals of the Rheumatic Diseases. 2014;73(6):968-74.

[35] Marras WS, Lavender SA, Ferguson SA, Splittstoesser RE, Yang G. Quantitative dynamic measures of physical exposure predict low back functional impairment. Spine. 2010;35(8):914-23.

[36] Burdorf A, van Riel M, Brand T. Physical load as risk factor for musculoskeletal complaints among tank terminal workers. American Industrial Hygiene Association Journal. 1997;58(7):489-97.

[37] Mundt DJ, Kelsey JL, Golden AL, Pastides H, Berg AT, Sklar J, et al. An epidemiologic study of non-occupational lifting as a risk factor for herniated lumbar intervertebral disc. The Northeast Collaborative Group on Low Back Pain. Spine. 1993;18(5):595-602.

[38] Nourollahi M, Afshari D, Dianat I. Awkward trunk postures and their relationship with low back pain in hospital nurses. Work. 2018;59(3):317-23.

[39] Callaghan JP, McGill SM. Intervertebral disc herniation: studies on a porcine model exposed to highly repetitive flexion/extension motion with compressive force. Clinical Biomechanics. 2001;16(1):28-37.

[40] Solomonow M. Neuromuscular manifestations of viscoelastic tissue degradation following high and low risk repetitive lumbar flexion. Journal of Electromyography and Kinesiology. 2012;22(2):155-75.

[41] Solomonow M, Zhou B-H, Baratta R, Lu Y, Harris $\mathrm{M}$. Biomechanics of increased exposure to lumbar injury caused by cyclic loading: Part 1 . Loss of reflexive muscular stabilization. Spine. 1999;24(23): 2426-34.

[42] Gunning JL, Callaghan JP, McGill SM. Spinal posture and prior loading history modulate compressive strength and type of failure in the spine: a biomechanical study using a porcine cervical spine model. Clinical Biomechanics. 2001;16(6):471-80

[43] McGill SM. Evolving ergonomics? Ergonomics. 2009;52(1):80-6.

[44] Fragala G, Bailey LP. Addressing occupational strains and sprains: Musculoskeletal injuries in hospitals. Aaohn Journal. 2003;51(6):252-9.

[45] Nelson AL. Safe patient handling and movement: A practical guide for health care professionals: Springer Publishing Company; 2005.

[46] Beach TA, Stankovic T, Carnegie DR, Micay R, Frost DM. Using verbal instructions to influence lifting mechanics-Does the directive "lift with your legs, not your back" attenuate spinal flexion? Journal of Electromyography and Kinesiology. 2018;38:1-6.

[47] Demoulin C, Marty M, Genevay S, Vanderthommen M, Mahieu G, Henrotin Y. Effectiveness of preventive back educational interventions for low back pain: a critical review of randomized controlled clinical trials. European Spine Journal. 2012;21(12):2520-30.

[48] Hignett S. Intervention strategies to reduce musculoskeletal injuries associated with handling patients: a systematic review. Occupational and Environmental Medicine. 2003;60(9):e6-e. 
[49] Morone G, Paolucci T, Alcuri M, Vulpiani M, Matano A, Bureca I, et al. Quality of life improved by multidisciplinary back school program in patients with chronic non-specific low back pain: a single blind randomized controlled trial. European Journal of Physical and Rehabilitation Medicine. 2011;47(4):533-41.

[50] Garcia AN, Gondo FL, Costa RA, Cyrillo FN, Silva TM, Costa LC, et al. Effectiveness of the back school and mckenzie techniques in patients with chronic non-specific low back pain: a protocol of a randomised controlled trial. BMC Musculoskeletal Disorders. 2011;12(1):179.

[51] Heymans MW, van Tulder MW, Esmail R, Bombardier C, Koes BW. Back schools for nonspecific low back pain: a systematic review within the framework of the Cochrane Collaboration Back Review Group. Spine. 2005;30(19):2153-63.

[52] Stubbs D, Buckle P, Hudson M, Rivers P, Worringham C. Back pain in the nursing profession I. Epidemiology and pilot methodology. Ergonomics. 1983;26(8):755-65.

[53] Chan V. A Comparison of Augmented Feedback and Didactic Approaches to Reduce Spine Motion During Box and Paramedic Lifting Tasks 2018.

[54] Contreras B, Schoenfeld B. To crunch or not to crunch: An evidence-based examination of spinal flexion exercises, their potential risks, and their applicability to program design. Strength \& Conditioning Journal. 2011;33(4):8-18.

[55] Caneiro J, O'Sullivan P, Lipp OV, Mitchinson L, Oeveraas N, Bhalvani P, et al. Evaluation of implicit associations between back posture and safety of bending and lifting in people without pain. Scandinavian Journal of Pain. 2018;18(4):719-28.

[56] O'Sullivan K. 2018 [Available from: https://www.inde pendent.ie/ca/life/health-wellbeing/everything-you-knowabout-back-pain-is-wrong-37797430.html.

[57] Videman T, Gibbons LE, Battié MC. Age-and pathology-specific measures of disc degeneration. Spine. 2008;33(25):2781-8.

[58] Caneiro J, O’Sullivan P, Smith A, Moseley GL, Lipp OV. Implicit evaluations and physiological threat responses in people with persistent low back pain and fear of bending. Scandinavian Journal of Pain. 2017;17:355-66.

[59] Seidler A, Euler U, Bolm-Audorff U, Ellegast R, Grifka J, Haerting J, et al. Physical workload and accelerated occurrence of lumbar spine diseases: risk and rate advancement periods in a German multicenter case-control study. Scandinavian Journal of Work, Environment \& Health. 2011:30-6.

[60] Marras WS, Lavender SA, Leurgans SE, Fathallah FA, Ferguson SA, Gary Allread W, et al. Biomechanical risk factors for occupationally related low back disorders. Ergonomics. 1995;38(2):377-410.

[61] Nelson A, Baptiste AS. Evidence-based practices for safe patient handling and movement. Clinical Reviews in Bone and Mineral Metabolism. 2006;4(1):55-69.

[62] Brookman C. The PSW Health and Safety Matters Survey. 2005.

[63] Beach TAC. Movement Assessment and (Re)Training for the Prevention and Management of Musculoskeletal Disorders- Strategies for "Occupational Athletes". CREMSD2016.

[64] Dozza M. Biofeedback Systems for Human Postural Control: a method for understanding sensory integration and improving motor training. Doctoral Thesis, University of Bologne, 2006.
[65] Azrin N, Rubin H, O'brien F, Ayllon T, Roll D. Behavioral engineering: Postural control by a portable operant apparatus. Journal of Applied Behavior Analysis. 1968;1(2):99-108.

[66] Wong M, Mak A, Luk K, Evans J, Brown B. Effectiveness of audio-biofeedback in postural training for adolescent idiopathic scoliosis patients. Prosthetics and Orthotics International. 2001;25(1):60-70.

[67] Lavender SA, editor A test of the lifttrainer: an aggressive approach for preventing back injuries through training. Proceedings of the Human Factors and Ergonomics Society Annual Meeting; 2000: SAGE Publications Sage CA: Los Angeles, CA.

[68] Mirelman A, Herman T, Nicolai S, Zijlstra A, Zijlstra W, Becker C, et al. Audio-biofeedback training for posture and balance in patients with Parkinson's disease. Journal of Neuroengineering and Rehabilitation. 2011;8(1):35.

[69] O'Sullivan K, O'Sullivan L, O'Sullivan P, Dankaerts W. Investigating the effect of real-time spinal postural biofeedback on seated discomfort in people with non-specific chronic low back pain. Ergonomics. 2013;56(8):1315-25.

[70] Kuipers DA, Wartena BO, Dijkstra BH, Terlouw G, van t Veer JT, van Dijk HW, et al. iLift: A health behavior change support system for lifting and transfer techniques to prevent lower-back injuries in healthcare. International Journal of Medical Informatics. 2016;96:11-23.

[71] O'Sullivan K, Verschueren S, Pans S, Smets D, Dekelver K, Dankaerts W. Validation of a novel spinal posture monitor: comparison with digital videofluoroscopy. European Spine Journal. 2012;21(12):2633-9.

[72] Intolo $\mathrm{P}$, Carman $\mathrm{AB}$, Milosavljevic S, Abbott JH, Baxter GD. The Spineange $\mathrm{l}^{\circledR}$ : Examining the validity and reliability of a novel clinical device for monitoring trunk motion. Manual Therapy. 2010;15(2):160-6.

[73] Lieberman J, Breazeal C. TIKL: Development of a wearable vibrotactile feedback suit for improved human motor learning. IEEE Transactions on Robotics. 2007;23(5):919-26.

[74] Ortega T, Ford D, Kajaks T, King E, Holyoke P, Prout E, Holliday P, Semple M, Longfield A, Dutta T. Pilot-testing PostureCoach: A wearable, real-time posture coaching system. Canadian Association of Occupational Therapists; April 19-22, 2016; Banff, AB, Canada 2016.

[75] Hélie S, Waldschmidt JG, Ashby FG. Automaticity in rule-based and information-integration categorization. Attention, Perception, \& Psychophysics. 2010;72(4): 1013-31.

[76] Hardwick RM, Forrence AD, Krakauer JW, Haith AM. Skill acquisition and habit formation as distinct effects of practice. 2017.

[77] Ashby FG, Ell SW, Waldron EM. Procedural learning in perceptual categorization. Memory \& Cognition. 2003;31(7):1114-25.

[78] Owlia M. Preventing back pain among caregivers using realtime movement-centered feedback (Masters dissertation).

[79] Dolan P, Mannion AF, Adams MA. Passive tissues help the back muscles to generate extensor moments during lifting. Journal of Biomechanics. 1994;27(8):1077-85.

[80] Kamachi M, Owlia M, Dutta T. Training Caregivers to Reduce Spine Flexion Using Biofeedback. International Conference on Applied Human Factors and Ergonomics 2019 Jul 24 (pp. 241-251). Springer, Cham. 